\title{
The Drosophila TNF Ortholog Eiger Is Required in the Fat Body for a Robust Immune Response
}

\author{
Eric M. Mabery David S. Schneider \\ Department of Microbiology and Immunology, Stanford University, Stanford, Calif., USA
}

\section{Key Words}

Drosophila $\cdot$ Salmonella $\cdot$ Tolerance $\cdot$ Resistance anorexia

\begin{abstract}
Eiger is the sole TNF family member found in Drosophila melanogaster. This signaling molecule is induced during infection and is required for an appropriate immune response to many microbes; however, little is known about where eiger is produced. Here, we show that eiger is made in the fly's fat body during a Salmonella typhimurium infection. Using tissue-specific knockdown, we found that eiger expression in the fat body is required for all of the phenotypes we observed in eiger null mutant flies. This includes reduced melanization, altered antimicrobial peptide expression and reduced feeding rates. The effect of eiger on feeding rates alone may account for the entire phenotype seen in eiger mutants infected with S. typhimurium.
\end{abstract}

Copyright $\odot 2010$ S. Karger AG, Basel (c) 2010 S. Karger AG, Basel

1662-811X/10/0024-0371\$26.00/0

Fax +41613061234 E-Mail karger@karger.ch www.karger.com

\section{Introduction}

Eiger is the only known TNF family member in Drosophila melanogaster $[1,2]$ and affects both resistance and tolerance to infections. Tolerance is defined as the slope resulting when survival is plotted against pathogen number $[3,4]$. Resistance is the ability of the host to clear pathogens $[3,4]$. Loss-of-function eiger mutants have 4 different phenotypes, depending upon which microbes are used to challenge the fly. The simplest phenotype is no change in tolerance or resistance. When nonpathogenic Escherichia coli is used to infect eiger mutants, the flies act like wild-type flies [5]. Salmonella typhimuriuminfected flies live longer than do wild-type flies but do not have increased bacterial growth, revealing an increase in tolerance with no change in resistance $[5,6]$. Listeria monocytogenes-infected flies show no change in survival but have a significant increase in bacterial levels, suggesting a balanced increase in tolerance and a decrease in resistance, resulting in no net change in defense [5]. Infections with Burkholderia cepacia or Streptococcus pneumoniae show decreased survival accompanied by increased bacterial growth [6]. This suggests a decrease in resistance and no changes in tolerance. 
Eiger regulates several physiological reactions important in fly immunity. Eiger appears to be a negative regulator of some antimicrobial peptides, as loss-of-function mutants have increased levels of transcripts encoding the antimicrobial peptide diptericin upon infection with several pathogens [5]. Eiger loss-of-function mutants show decreased phagocytic activity towards Staphylococcus aureus, demonstrating that eiger is required for hemocyte function [5]. Eiger has also been implicated in melanization responses in larvae and is needed for proper crystal cell function [7]. How these physiological reactions work together to influence resistance and tolerance during an infection is not understood.

To determine where eiger affects tolerance, we infected flies with S. typhimurium and monitored the tissue-specific requirements for eiger function. We started by determining where eiger was induced during an infection and found that it was expressed in the fat body. Given this expression pattern and the defect in hemocyte activity seen in eiger null mutants, we proceeded to test eiger function in these two tissues. We found that knocking down eiger expression in the fat body led to an increase in survival but pathogen loads did not change, i.e. an increase in tolerance with no change in resistance. Eiger knockdown in the fat body also caused an increase in Imd-mediated AMP transcription and a decrease in melanization.

\section{Materials and Methods}

\section{Survival Assay}

Overnight cultures of S. typhimurium (SL1344) were grown in Luria broth, unshaken, at $37^{\circ} \mathrm{C}$. Using a drawn glass capillary, 50 $\mathrm{nl}$ of medium (containing 10,000 bacteria) was injected into 5 - to 7-day-old age-matched male flies on their ventrolateral abdomen using a Picospritzer 3 apparatus [6]. Three sets of 20 flies were injected for each condition in an experiment, and each whole experiment was repeated at least 3 times. Treated flies were incubated at $29^{\circ} \mathrm{C}$ in a 12 -hour/12-hour light/dark incubator at $65 \%$ relative humidity following injection and fed dextrose food (129.4 $\mathrm{g}$ of dextrose, $7.4 \mathrm{~g}$ of agar, $61.2 \mathrm{~g}$ of cornmeal, $32.4 \mathrm{~g}$ of yeast and $2.7 \mathrm{~g}$ of Tegosept with water added to make up 1 liter). Survival was monitored daily. To control for background effects and to test for leaky expression of the RNAi construct, we crossed all flies containing constructs (the eiger knockdown construct and the Gal4 flies) to the $\mathrm{w}^{1118}$ wild type. Kaplan-Meier survival curves were generated using Graphpad Prism. Log-rank analysis was performed with Graphpad Prism.

\section{Bacterial Counts}

A total of 10,000 bacteria were injected into 5- to 7-day-old age-matched male flies as described above. At least 3 flies for each time point were homogenized individually in $\mathrm{PBS}(\mathrm{pH}=7.5)$. The homogenate was further diluted in PBS and then plated onto Luria broth agar plates. Plates were incubated overnight at $37^{\circ} \mathrm{C}$.
Statistical significance was calculated using ANOVA with Graphpad Prism.

\section{Melanization Assay}

S. typhimurium was used in all infections. A total of 10,000 bacteria were injected into 5- to 7-day-old age-matched male flies. Infected flies were incubated for 6 days at $29^{\circ} \mathrm{C}$. After 6 days, the melanized spots on the flies were counted using a dissecting microscope. $\chi^{2}$ analysis was performed to determine statistical significance using Graphpad Prism.

\section{Quantitative RT-PCR Assay of Antimicrobial Peptides and} Eiger Transcripts

RNA was isolated using TRIzol reagent. Three groups of 5 flies were tested for each time point, and each experiment was performed at least 3 times. RNA samples were treated with DNase then quantified by quantitative RT-PCR (qRT-PCR) reactions. Each reaction was performed in triplicate using the Sybr Green One Step RT-PCR kit from Qiagen using the BioRad icycler. Primers used were: drosomycin primers, 5'-GACTTGTTCGCCCTCTTCG-3' and 5'-CTTGCACACAGACGACAG-3'; diptericin primers, 5'-ACCGCAGTACCCACTCAATC-3' and $5^{\prime}$-CCCAAGTGCTGTCCATATCC-3'; ;igerprimers, $5^{\prime}$-GATGGTCTGGATTCCATTGC-3' and 5'-TAGTCTGCGCCAACATCATC-3'; eiger nri primers, $5^{\prime}$-CTGCCGAGACCCTCAAGC-3' and $5^{\prime}$ AGATCGTTAGTGCGAGAATG-3'. Data are reported as the level of AMP divided by the level of ribosomal protein 15a, to normalize the data to a housekeeping gene. ANOVA was performed using Graphpad Prism.

\section{Feeding Assay}

For this assay, 5- to 7-day-old age-matched male flies were injected with 10,000 bacteria. Three groups of 20 flies were used, and these experiments were performed at least 3 times. Injected flies were incubated for $24 \mathrm{~h}$ at $29^{\circ} \mathrm{C}$. These flies were then given fly food containing $0.5 \%$ xylene cyanol and $0.1 \%$ bromophenol blue. After an initial 5-min measurement, ingestion rates were measured at 15-min intervals by counting flies that had blue abdomens. Total ingestion was measured by homogenizing at least 10 flies in $200 \mu \mathrm{l}$ of $10 \mathrm{~mm}$ Tris and $1 \mathrm{~mm}$ EDTA, $\mathrm{pH}$ 7.5, at various time points in the linear phase of ingestion. To remove fly parts, the solution was then centrifuged for $5 \mathrm{~min}$ at 13,000 rpm. Then $800 \mu \mathrm{l}$ of $10 \mathrm{~mm}$ Tris and $1 \mathrm{~mm}$ EDTA, pH 7.5, was added to the solution, and absorbance (optical density) at $614 \mathrm{~nm}$ was used to quantify total dye ingested.

\section{Eiger Gene Expression Reporter}

The 2-kb region upstream of eiger was amplified using the following primers: 5'-AGTGTGCGAATCAATCCACAGTAAAC-3' (forward) and 5'-AATTTGAACTGAGAACGGGAGCGTG-3' (reverse). This PCR product was then subcloned into the Topo XL vector (Invitrogen). After digestion with EcoRI, the eiger promoter was then ligated into the PPTGAL vector [8]. Sequencing was used to verify cloning. Transgenic flies were generated by BestGene Inc. (Chula Vista). The transgenic flies were then crossed to UAS-green fluorescent protein (GFP) flies. The F1 progeny from this cross were used in these experiments. At least 5 age-matched flies were injected with 10,000 bacteria and incubated at $29^{\circ} \mathrm{C}$. GFP expression was monitored using a Leica MZ3 fluorescent dissecting scope. Images were taken before injection and $72 \mathrm{~h}$ after injection. 


\section{Results and Discussion}

Eiger Transcription Is Activated in the Fat Body during S. typhimurium Infection

Past work demonstrated a 5- to 8-fold increase in eiger transcript accumulation during $S$. typhimurium infection; however, the location of eiger expression remained unknown [5]. Understanding which tissue produces eiger could give us insight into how eiger functions. To determine which tissue produces eiger, we cloned the $2-\mathrm{kb}$ region upstream of the predicted start site for eiger into a vector that drives the expression of Gal4 in an eiger promoter-dependent fashion. We made transgenic flies containing this construct and crossed them to flies containing UAS-GFP, which allows us to monitor eiger promoter activity by following GFP expression. We used the F1 progeny to study eiger transcription. Initially, we used qRT-PCR to monitor eiger transcription in adult flies after S. typhimurium infection and found that a 5- to 8-fold increase in eiger transcript levels occurs $72 \mathrm{~h}$ after infection (fig. 1). To determine eiger transcription patterns in adult flies during an infection, we visually monitored the expression of GFP in flies before and after S. typhimurium infection. Before infection, GFP expression was localized to the fat body, and $72 \mathrm{~h}$ after infection, GFP fluorescence grew more intense in the fat body (fig. 2). Localization to the fat body is difficult to visualize in the whole fly because the fat body is attached to the wall of the abdominal cavity and is hard to separate from other tissue. To address this issue, we filleted open the abdomen, exposing the inner surface of the fat body (fig. 2). We did not observe GFP expression in the hemocytes at any time.

Eiger Expression in the Fat Body Accounts for Most of the Eiger Immune Phenotype

We decided to knock down eiger expression in the fat body and in hemocytes. We chose the fat body because it produces many immune effectors and our eiger expression studies show that it is responsive to infection. We chose hemocytes because we know from past work that eiger affects hemocyte function, although we did not observe GFP expression in hemocytes using our eiger promoter construct [5].

We crossed flies containing tissue-specific Gal4 drivers (hemolectin, a hemocyte-specific protein, for hemocytes and larval serum protein 2 , a fat body-specific protein, for the fat body) $[9,10]$ to flies carrying an eiger RNAi construct [1]. To determine whether eiger was knocked down during these treatments, we used qRTPCR to monitor eiger mRNA levels $24 \mathrm{~h}$ after $S$. ty-

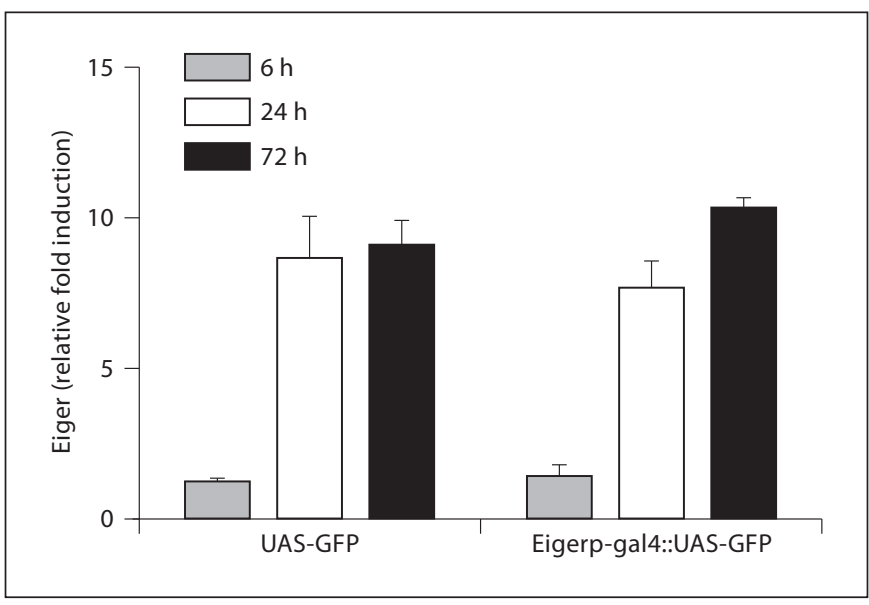

Fig. 1. Eiger induction during an S. typhimurium infection. Eiger mRNA was quantified 6, 24 and $72 \mathrm{~h}$ after infection. Transcript levels were normalized to ribosomal protein 15a transcripts, which are not altered during infection, and induction was normalized to levels found in unmanipulated flies. UAS-GFP = Control flies containing GFP; Eigerp-gal4::UAS-GFP = eiger promoter-driven expression of GFP. Error bars display the standard error of the mean.

phimurium infection. We found that eiger induction was reduced in all flies that contained the UAS-eiger inverted repeats (IR) construct and propose that this is due to leaky expression of the UAS-eiger IR construct. However, eiger induction is almost undetectable when eiger is knocked down in the fat body, and the decrease is significant when compared to UAS-eiger IR flies (fig. 3). After confirming knockdown, we performed survival assays on tissue-specific eiger knockdown flies. We infected these flies with $S$. typhimurium and monitored survival. Knockdown of eiger in the fat body significantly increased survival time during an S. typhimurium infection (table 1). Our past work also demonstrated that eiger null mutants survive $S$. typhimurium infections longer than do wild-type flies. This new work supports and strengthens our past work by demonstrating a statistically significant eiger phenotype in a different genetic background using RNAi to knock down eiger function rather than null alleles. These new survival data, combined with the eiger promoter transcription data, imply that the fat body is a key organ for the production of eiger. Eiger knockdown in hemocytes did not produce a statistically significant result.

We previously reported that eiger null mutants infected with S. typhimurium maintained relatively constant levels of bacteria that were indistinguishable from those 

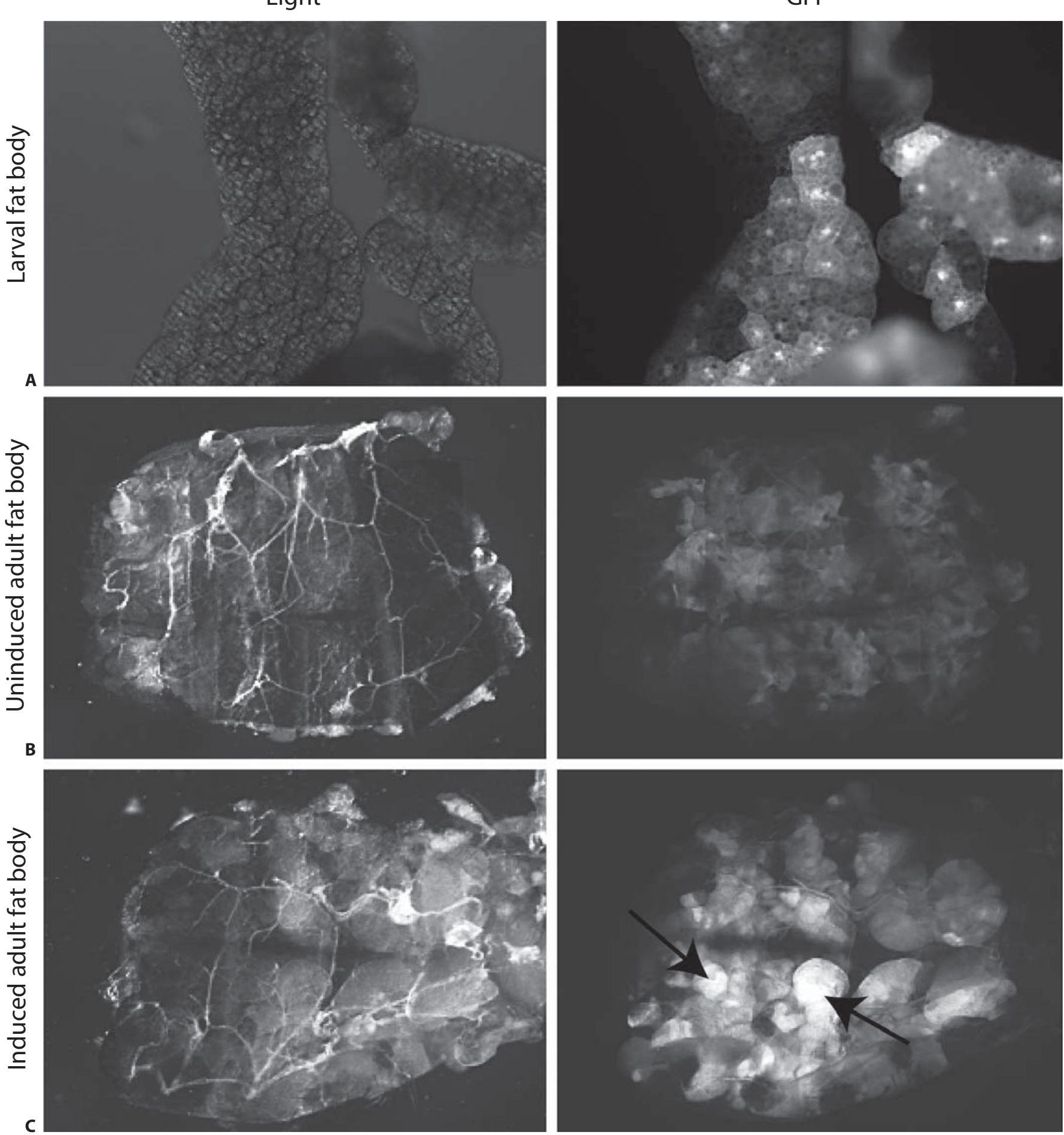

Fig. 2. Eiger promotor-driven expression of GFP. Dissected organs are shown under white light and GFP epifluorescence illumination. A Uninduced fat body dissected from a 3rd instar larva; some cells with an active eiger promotor can be seen in this uninduced tissue. B A filleted abdomen is presented inside up, revealing the fat body. Some induced cells can be seen in this uninduced fly. C A filleted abdomen from a S. typhimuriuminfected fly $72 \mathrm{~h}$ after infection. Arrows point to high levels of GFP expression. 


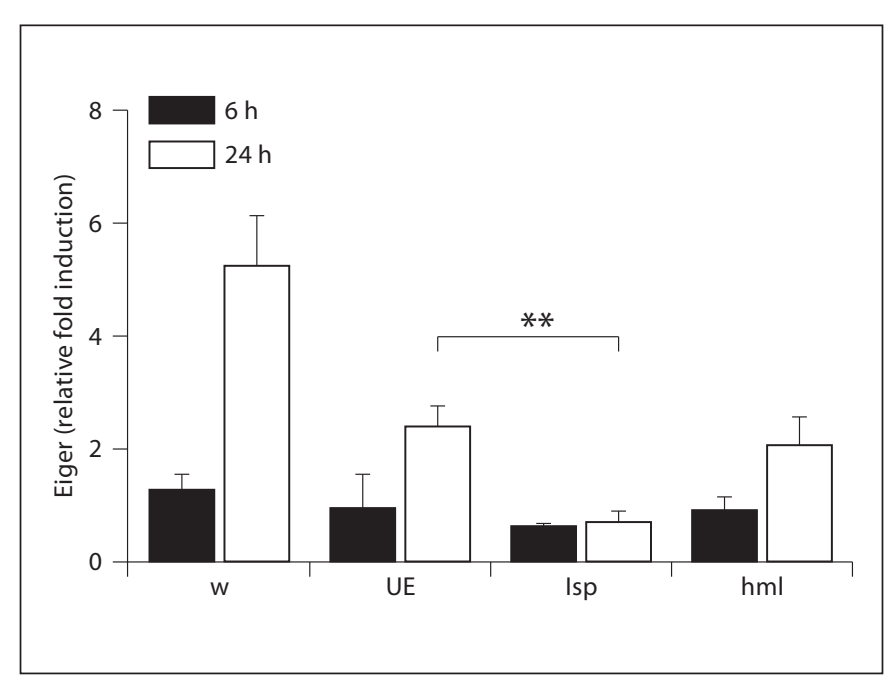

Fig. 3. Eiger induction in tissue-specific knockdown flies. Eiger mRNA levels were quantified 6 and $24 \mathrm{~h}$ after infection. Transcript levels were normalized to ribosomal protein 15a transcripts, which are not altered during infection, and induction was normalized to levels found in unmanipulated flies. $\mathrm{w}=\mathrm{w}^{1118}$ (isogenic wild type); UE = UAS-eiger IR (background flies that generate eiger RNAi after gal4 activation); lsp = larval serum protein 2-gal4::UAS-eiger IR (fat body knockdown flies); hml = hemolectin-gal::UAS-eiger IR (hemocyte knockdown flies). ANOVA and a post hoc Tukey test were used to test for significance. ${ }^{* *} \mathrm{p}<0.01$.

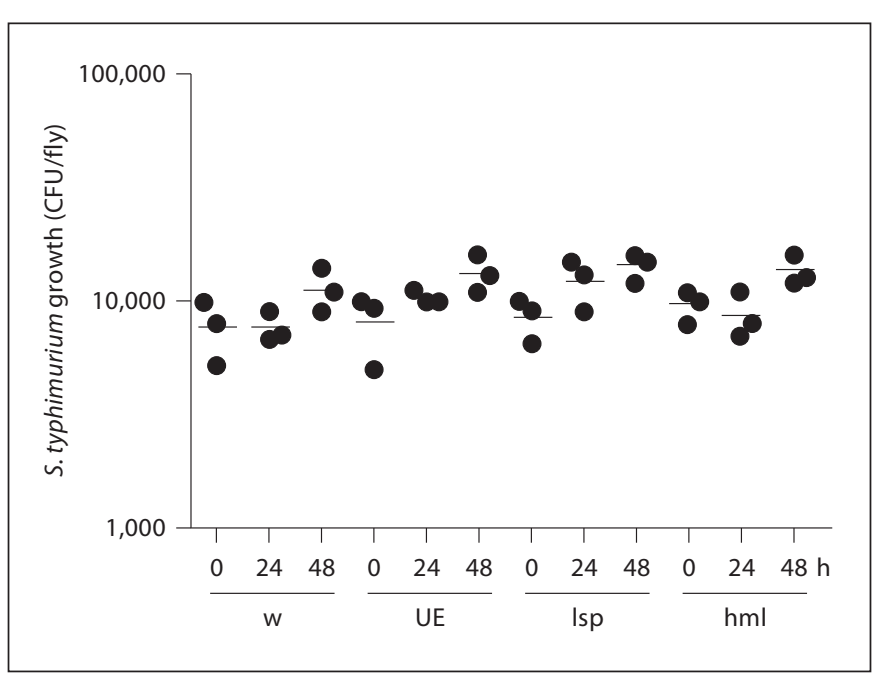

Fig. 4. The effect of eiger knockdown on the growth of $S$. $t y$ phimurium in infected flies. Flies were infected with S. typhimuri$u m$ and bacterial growth was monitored 0,24 and $48 \mathrm{~h}$ after infection. $\mathrm{w}=\mathrm{w}^{1118}$ (isogenic wild type); UE = UAS-eiger IR (background flies that generate eiger RNAi after gal4 activation); lsp = larval serum protein 2-gal4::UAS-eiger IR (fat body knockdown flies); hml = hemolectin-gal::UAS-eiger IR (hemocyte knockdown flies). Horizontal bars indicate means. Unpaired two-tailed $\mathrm{t}$ tests showed no statistically significant differences between flies given different treatments. found in wild-type flies $[5,6]$. To determine whether this was the case with RNAi knockdown of eiger, we injected S. typhimurium into the tissue-specific eiger knockdown flies and monitored bacterial growth over a 48-hour time period. We observed that bacterial loads were unchanged over the course of the experiment (fig. 4). These results taken together with the survival results imply that tissuespecific knockdown of eiger in the fat body alters tolerance and not resistance. Again, this supports our past results with eiger null mutants $[5,6]$.

Activation of the two known microbe-sensing pathways (Toll and Imd pathways) leads to production of antimicrobial peptides in the fat body [11]. As stated, eiger null mutants have increased levels of some Imd-induced antimicrobial peptides during infection. To determine which tissue was responsible for the changes in AMP expression, we used qRT-PCR to monitor the transcription of an antimicrobial peptide mainly induced by the Toll pathway (drosomycin) and one mainly induced by the Imd pathway (diptericin) during a $S$. typhimurium infection. S. typhimurium is a strong inducer of Imd signaling because it contains diaminopimelic acid-type peptidoglycan. We found that when eiger is knocked down in the
Table 1. Survival of tissue-specific eiger knockdown flies during a S. typhimurium infection

\begin{tabular}{|c|c|c|}
\hline & $\begin{array}{l}\text { Mean time } \\
\text { to death days }\end{array}$ & $\mathrm{p}$ value \\
\hline UAS-eiger IR & 14.5 & - \\
\hline $\mathrm{w}^{118}$ & 13 & 0.077 \\
\hline lsp-gal4::UAS-eiger IR & 21 & 0.0001 \\
\hline lsp-gal4:: $\mathrm{w}^{118}$ & 14 & 0.12 \\
\hline hml-gal4::UAS-eiger IR & 10 & 0.08 \\
\hline hml-gal4:: $\mathrm{w}^{118}$ & 12.5 & 0.095 \\
\hline
\end{tabular}

Flies were injected with S. typhimurium and survival was monitored daily. Statistical significance was determined using log rank analysis.

$\mathrm{p}$ values were calculated in comparison to UAS-eiger IR.

fat body, these flies had higher levels (approx. 3-fold increase; $p<0.01$ ) of diptericin but showed no change in drosomycin induction. When eiger was knocked down in the hemocytes, there was no change in induction of drosomycin and diptericin compared to control flies (fig. 5). 


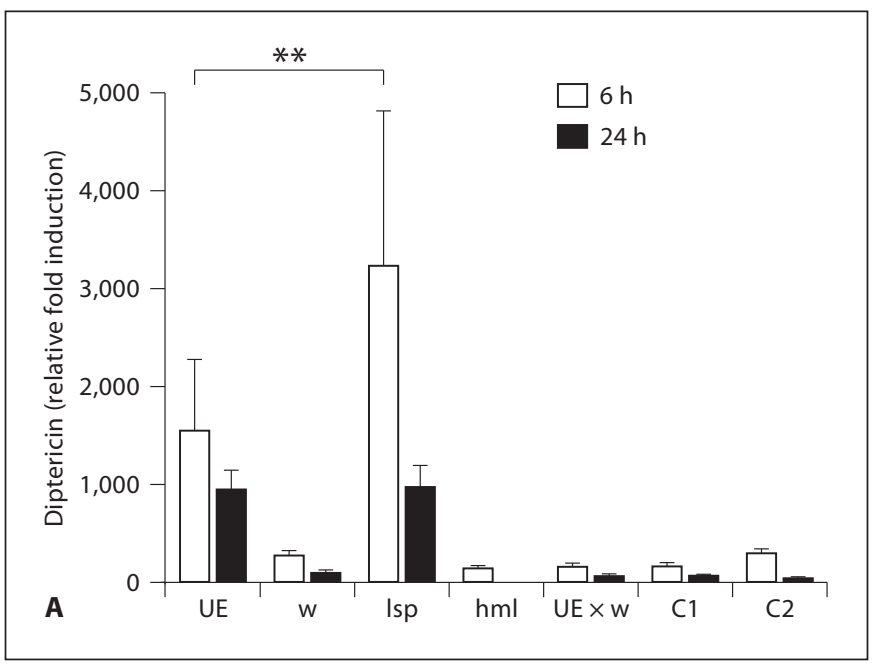

Fig. 5. Effect of eiger knockdown on antimicrobial peptide expression during infection. Flies were infected with S. typhimurium. mRNAs of the antimicrobial peptides diptericin (A) and drosomycin (B) were quantified 6 and $24 \mathrm{~h}$ after infection. Transcript levels were normalized to ribosomal protein 15a transcripts, which are not altered during infection, and induction was normalized to levels found in unmanipulated flies. $\mathrm{w}=\mathrm{w}^{1118}$ (iso-

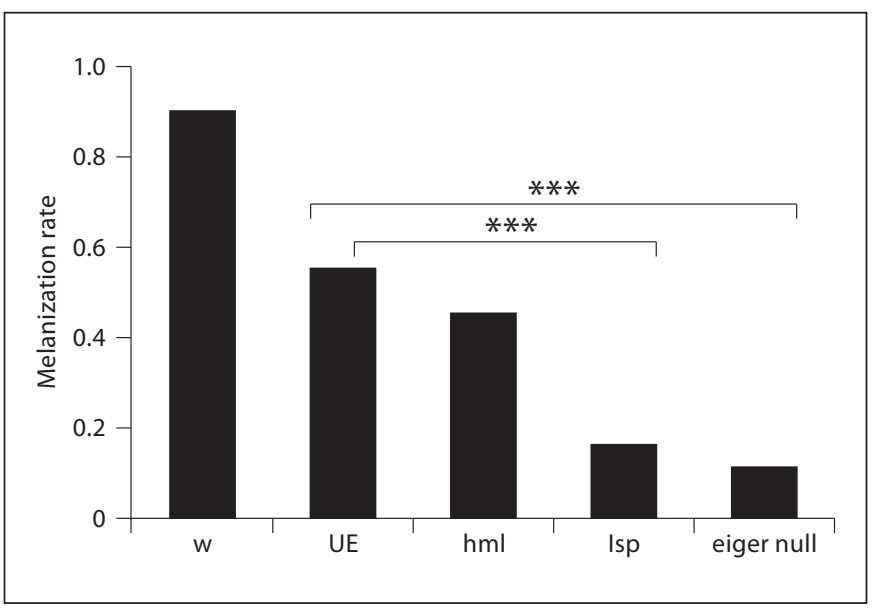

Fig. 6. The effect of eiger knockdown on melanization. Flies were infected with S. typhimurium. Melanization was scored visually 6 days after infection. $\mathrm{w}=\mathrm{w}^{1118}$ (isogenic wild type); UE $=$ UASeiger IR (background flies that generate eiger RNAi after gal4 activation); lsp = larval serum protein 2-gal4::UAS-eiger IR (fat body knockdown flies); hml = hemolectin-gal::UAS-eiger IR (hemocyte knockdown flies); eiger null $=$ eiger $^{1} \times$ eiger $^{3}\left(\right.$ eiger $^{1}$ and eiger $^{3}$ are different null alleles of eiger). $\chi^{2}$ analysis was used to test for statistical significance. ${ }^{* * *} \mathrm{p}<0.001$.

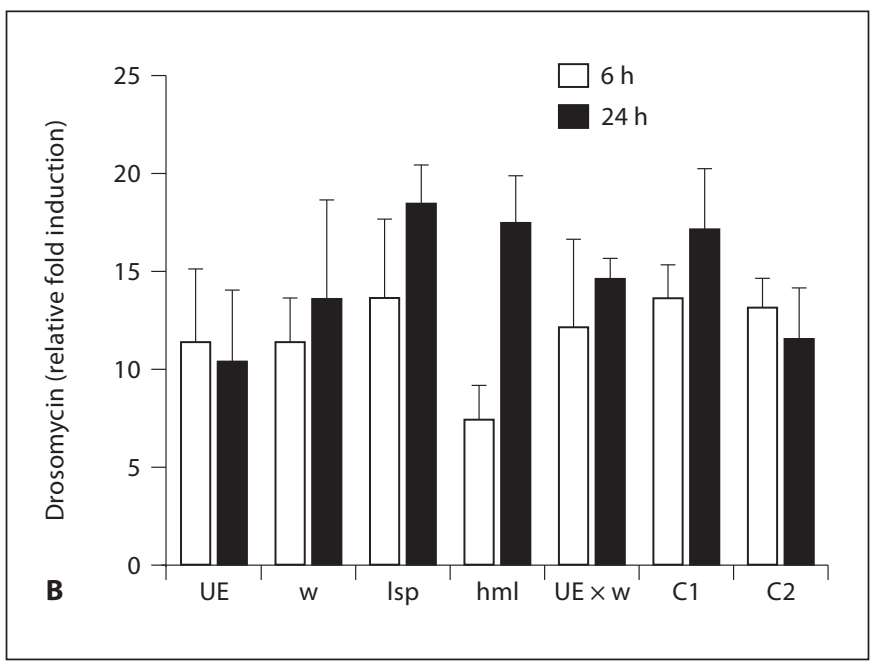

genic wild type); UE = UAS-eiger IR (background flies that generate eiger RNAi after gal4 activation); lsp = larval serum protein 2-gal4::UAS-eiger IR (fat body knockdown flies); hml = hemolectin-gal::UAS-eiger IR (hemocyte knockdown flies); UE $\times \mathrm{W}=$ UAS-eiger IR::: $\mathrm{w}^{1118} ; \mathrm{C} 1=$ lsp-gal4:: $\mathrm{w}^{1118} ; \mathrm{C} 2=$ hml-gal4:: $\mathrm{w}^{1118}$ (all background control crosses). ANOVA was performed to determine statistical significance. ${ }^{* *} \mathrm{p}<0.01$.

\section{Eiger and Melanization}

The melanization reaction is a complex host immune response that leads to the sequestration and death of invading pathogens [7]. This reaction depends on the activation of prophenoloxidase. Phenoloxidase initiates the melanization reaction by catalyzing the conversion of tyrosine to 3,4-dihydroxyphenylalanine. The end result of this reaction is the cross-linking of melanin around the pathogen and the production of reactive oxygen [7].

Ayres and Schneider [12] showed that melanization is important in fighting bacterial infections in the fly, in contrast to what has been previously reported [13]. They infected a fly line that is unable to melanize with a panel of pathogens. They monitored survival and pathogen growth and found that knocking out melanization decreases resistance to L. monocytogenes and S. typhimurium [12]. With this in mind, we measured melanization in eiger knockdown flies after $S$. typhimurium infection. We found that melanization was reduced in all tissue-specific knockdown flies when compared to wild-type flies, although the most severe melanization defects were seen with eiger knockdown in the fat body (fig. 6). This is an interesting result because Ayres and Schneider [12] showed that melanization is required to fight $S$. typhimurium infections (i.e. in the absence of melanization, flies infected with S. typhimurium do not survive as long 


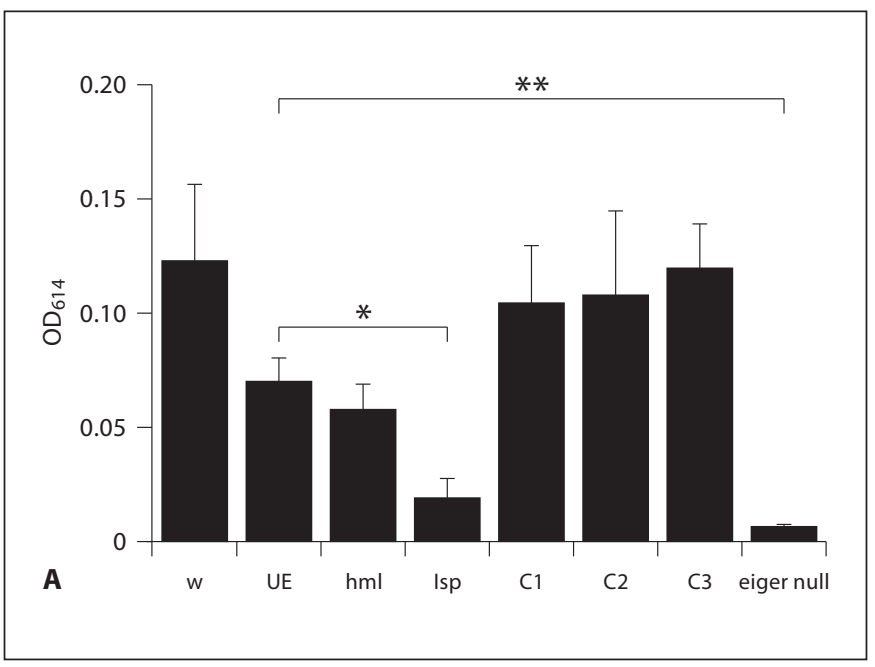

Fig. 7. Feeding rates in tissue-specific eiger knockdown flies during an S. typhimurium infection. Flies were infected with $S$. typhimurium (A) or were not manipulated (B). Total meal volumes were measured. Optical density (OD) at $614 \mathrm{~nm}$ was used to quantify total dye ingested. Feeding rates were determined for each set of flies and then time points were selected that corresponded to nonsaturating feeding rates. $\mathrm{w}=\mathrm{w}^{1118}$ (isogenic wild type); $\mathrm{UE}=$ UAS-eiger IR (background flies that generate eiger RNAi after

and bacterial growth is greater). If eiger were simply altering melanization during a S. typhimurium infection, then these flies should show a decrease in resistance; however, we observed an increase in tolerance, which suggests that the role of eiger is more complicated then being a simple regulator of phenoloxidase. An alternative explanation is that the visible tissue damage seen from melanization is not directly correlated with phenoloxidase activity; therefore, the lack of melanization spots seen in the absence of eiger could be due to other factors related to tissue damage.

\section{Eiger Knockdown Alters Feeding Rates}

We previously observed a constellation of phenotypes similar to this eiger effect; flies carrying a mutation in the taste receptor gr28b do not melanize, have an increased tolerance to $S$. typhimurium and have decreased melanization [14]. We found that the explanation for this phenotype is that the flies consume less food than do wild-type flies, and the phenotype could be reproduced simply by restricting the flies' diet. This suggested that a simple explanation for the eiger phenotype could be that these flies also eat less than wild-type flies.

To determine if eiger knockdown flies have reduced feeding rates, we infected tissue-specific eiger knock-

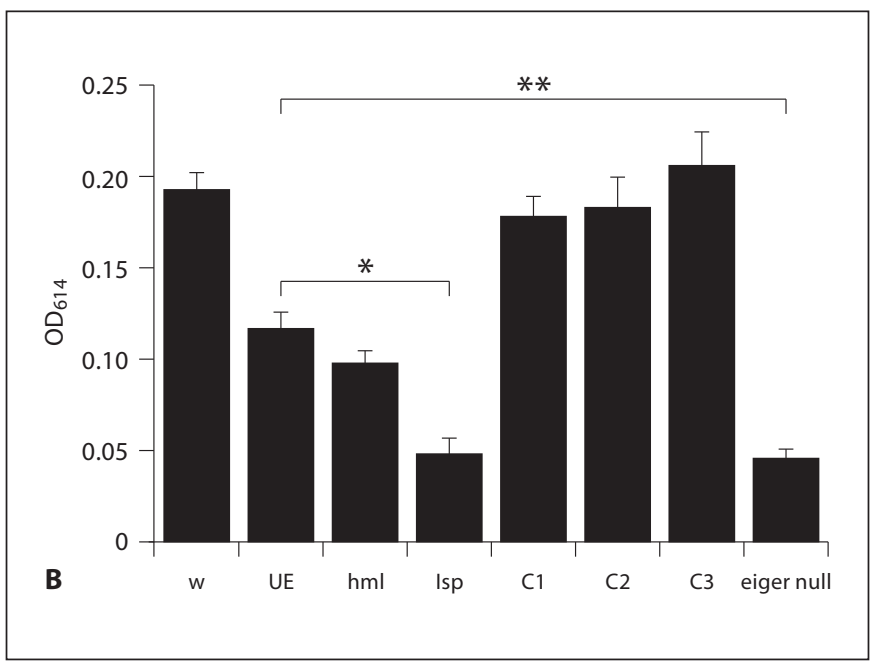

gal4 activation); lsp = larval serum protein 2-gal4::UAS-eiger IR (fat body knockdown flies); hml = hemolectin-gal::UAS-eiger IR (hemocyte knockdown flies); C1 = lsp-gal4:: $\mathrm{w}^{1118}$; C2 = hmlgal4:: $\mathrm{w}^{1118}$; C3 = UAS-eiger:: $\mathrm{w}^{1118}$ (all background control crosses); eiger null $=$ eiger $^{1} \times$ eiger $^{3}$ (eiger ${ }^{1}$ and eiger $^{3}$ are different null alleles of eiger). ANOVA analysis was used to test for statistical significance. ${ }^{* *} \mathrm{p}<0.01$.

down flies with S. typhimurium and $24 \mathrm{~h}$ later measured rates of ingestion. We found that knockdown of eiger reduced feeding rates compared to wild-type and background controls (fig. 7). This change in feeding rate may be responsible for some aspects of the eiger phenotype, although it must be noted that even in the absence of infection, eiger knockdown flies consume less food (fig. 7). The reduction in feeding seen in infected flies with eiger knocked down in the fat body was statistically significant $(p<0.05)$ when compared to the uninfected flies with eiger fat body knockdown.

In summary, the majority of the eiger-regulated interactions between the fly and S. typhimurium appear to require eiger expression in the fat body. Expression in the fat body can explain survival, bacterial growth and antimicrobial peptide gene expression phenotypes. These results fully support and strengthen our past publications by reproducing these eiger phenotypes in different genetic backgrounds using different types of mutants. The story is complicated, however, because flies with low levels of eiger expression eat less. This change in feeding rate could also explain the phenotype of eiger mutants with respect to $S$. typhimurium infection because these eiger phenotypes resemble those of diet-restricted flies. Reduced feeding alone may explain the interaction of an 
eiger mutant fly with S.typhimurium but does not explain the whole phenotype; for example, we showed previously that reduced feeding decreases resistance to $L$. monocytogenes, but an eiger null mutant shows a balanced decrease in resistance and an increase in tolerance to L. monocytogenes infections [14]. Similar to what has now been observed with regards to melanization and feeding, eiger too has complex effects on the fly's immune response.

\section{References}

1 Igaki T, Kanda H, Yamamoto-Goto Y, Kanuka $\mathrm{H}$, Kuranaga E, Aigaki T, Miura M: Eiger, a TNF superfamily ligand that triggers the Drosophila JNK pathway. EMBO J 2002; 21:3009-3018.

$\checkmark 2$ Kauppila S, Maaty WS, Chen P, Tomar RS, Eby MT, Chapo J, Chew S, Rathore N, Zachariah S, Sinha SK, Abrams JM, Chaudhary PM: Eiger and its receptor, Wengen, comprise a TNF-like system in Drosophila. Oncogene 2003;22:4860-4867.

3 Raberg L, Sim D, Read A: Disentangling genetic variation for resistance and tolerance to infectious diseases in animals. Science 2007; 318:812-814.

4 Schneider DS, Ayres JS: Two ways to survive infection: what resistance and tolerance can teach us about treating infectious diseases. Nat Rev Immunol 2008;8:1474-1733.

5 Schneider DS, Ayres JS, Brandt SM, Costa A, Dionne MS, Mabery E, Moule M, ShirasuHiza M: Drosophila eiger mutants are sensitive to extracellular pathogens. PLoS Pathog 2007;3:e41.
- 6 Brandt SM, Dionne MS, Khush RS, Pham LN, Vigdal TJ, Schneider, D: Secreted bacterial effectors and host-produced eiger/TNF drive death in a Salmonella-infected fruit fly. PLoS Biol 2004;2:e418.

7 Bidla G, Dushay MS, Theopold U: Crystal cell rupture after injury in Drosophila requires the JNK pathway, small GTPases and the TNF homolog Eiger. J Cell Sci 2007;120: 1209-1215.

8 Sharma Y, Cheung U, Larsen EW, Eberl DF: pPTGAL, a convenient Gal4 P-element vector for testing expression of enhancer fragments in drosophila. Genesis 2002;34:115118.

-9 Goto A, Kadowaki T, Kitagawa Y: Drosophila hemolectin gene is expressed in embryonic and larval hemocytes and its knockdown causes bleeding defects. Dev Biol 2003;264: 582-591.
10 Benes H, Neal KC, Willis RL, Gadde D, Castleberry $\mathrm{AB}$, Korochkina SE: Overlapping Lsp-2 gene sequences target expression to both the larval and adult Drosophila fat body. Insect Mol Biol 1996;5:39-49.

11 Ferrandon D, Imler JL, Hetru C, Hoffmann JA: The Drosophila systemic immune response: sensing and signaling during bacterial and fungal infections. Nat Rev Immunol 2007;7:862-874.

12 Ayres JS, Schneider DS: A signaling protease required for melanization in Drosophila affects resistance and tolerance of infections. PLoS Biol 2008;6:e305.

13 Leclerc V, Pelte N, El Chamy L, Martinelli C, Ligoxygakis P, Hoffmann JA, Reichhart JM: Prophenoloxidase activation is not required for survival to microbial infections in Drosophila. EMBO 2007;7:231-235.

14 Ayres JS, Schneider DS: The role of anorexia in resistance and tolerance to infections in Drosophila. PLoS Biol 2009;7:e1000150. 